\title{
IN PURSUIT OF AN OPTIMUM OPTICAL IMAGING TECHNOLOGY FOR EARLY DETECTION OF DENTAL CARIES
}

\author{
Elnaz Baradaran Shokouhi \\ Department of Mechanical Engineering \\ York University, Hybrid Biomedical Optics Lab \\ 4700 Keele Street, Toronto, Ontario M3J 1P3, Canada \\ ebaradar@yorku.ca
}

\author{
Nima Tabatabaei \\ Department of Mechanical Engineering \\ York University, Hybrid Biomedical Optics Lab \\ 4700 Keele Street, Toronto, Ontario M3J 1P3, Canada \\ Nima.Tabatabaei@lassonde.yorku.ca
}

\begin{abstract}
In the last two decades, majority of the newly developed dental caries detection techniques have been opticsbased, relying either on enhancement of light scattering in early carious lesion (e.g. optical coherent tomography or OCT) or enhancement of light absorption in early caries (e.g. thermophotonic lock-in imaging or TPLI). This paper aims to explore the detection threshold capabilities between light scattering and light absorption based dental caries detection methods. With this intention, the experiments will be conducted through examination of controlled artificiallyinduced early caries. It is anticipated that the molecularcontrast TPLI imaging technology outperforms OCT due to the more specific nature of light absorption contrast mechanism.
\end{abstract}

Keywords-component; early dental caries; thermophotonic lock-in imaging; spectral-domain optical coherence tomography; artificial demineralization.

\section{INTRODUCTION}

Dental caries continues to be a major public health challenge in both children and adults [1]. Over the past years, the state of the art in preventive dentistry has provided promising therapeutic techniques for preventing the progression of early dental caries to cavities and complete tooth decay [2]. However, the effectiveness of these preventive provisions rely on detection of caries at very early stages. But, unfortunately, clinical detection methods such as x-ray radiography and visual-tactile inspection lack sufficient sensitivity for detection of early stages of carious legions due to morphology of tooth structure and large surface area of healthy enamel around the carious legion [3]. In this paper, a quantification of detection threshold for mainstream optical imaging modalities, optical coherence tomography (OCT) and thermophotonic lock-in imaging (TPLI) (as representative techniques based on light scattering and absorption, respectively), is performed in early detection of dental caries. Optical coherence tomography (OCT) is a promising modality capable of imaging samples based on back reflection of light, and is capable of providing 3-dimensional sub-surface morphology of biological tissue microstructure with micronscale resolution [4]. The ability of OCT in providing high- resolution 3-dimensional images has led to its popularity in dentistry especially in diagnosis and screening of dental diseases [5][6].

While OCT provides three dimensional maps of lightreflecting interfaces in biological tissues, such as void interfaces introduced by demineralization in tooth, light absorption-based technologies, such as TPLI, produce maps of subsurface features absorbing the specific incident laser radiation (e.g., Calcium and phosphate as the byproducts of demineralization). The working principle of lock-in thermography involves an incorporation of active thermography along with lock-in demodulation signal processing in turbid samples to detect thermal waves emitted back from subsurface molecular absorption sites (e.g. early dental caries) by focusing the infrared camera on the sample [7]. As such, lock-in thermography is a very advantageous tool in electronic device testing and failure analysis of materials [8], but it also has important applications in early pre-clinical diagnosis and control during treatment in medical fields such as imaging of early caries [9]. Therefore, the motivation behind this study is to evaluate the detection threshold of two emerging medical imaging technologies, spectral domain-OCT (OCT) and TPLI, in diagnosis of early dental caries.

\section{MATERIALS AND METHODS}

\section{A. Thermophotonic Lock-in Imaging}

The experimental setup of TPLI (Fig. 1(a)) [10] consists of a continuous wave $808 \mathrm{~nm}$ near-infrared laser diode $(808 \mathrm{~nm}$; Jenoptik, Jena, Germany) which is regulated by a laser controller unit (Ostech, Berlin, Germany) in order to allow for modulation of laser intensity. Samples are put on LEGO blocks and positioned at the focal plane of the camera. The LWIR camera (Gobi 640; Xenics, Leuven, Belgium) used has a spectral range of $(8-14 \mu \mathrm{m})$ and maximum frame rate of 50 frames per second. The detector of the camera consists of $640 \times 480$ elements with pixel pitch of $25 \mu \mathrm{m}$. A custom made extension tube and an $18 \mathrm{~mm}$ focal-length objective lens are used to obtain a magnification of one from the interrogated surface of the sample.

The multifunctional data acquisition board synchronously generates three signals: reference pulse train, in-phase, and 
quadrature reference signals. The reference pulse train finds the beginning of each modulation cycle for averaging. The inphase reference signal is also sent to the laser controller to modulate the intensity of the laser beam. The in-phase reference signal is in-phase with the laser modulation while there is a $90^{\circ}$ phase lead between the in-phase and quadrature reference signals. The images are then taken with a long-wave infrared camera and then weighed and averaged using the instantaneous readings of the two reference signals to yield inphase $\left(\mathrm{S}^{0}\right)$ and quadrate $\left(\mathrm{S}^{90}\right)$ images [10]. The amplitude and phase for each pixel are then calculated by applying equation 1 :

$$
\mathrm{A}=\left[\left(S^{0}\right)^{2}+\left(S^{90}\right)^{2}\right]^{0.5} \text { and } \varphi=\arctan \left(S^{90} / S^{0}\right)
$$

In this paper, we only present and discuss the phase images as they are known to be of superior sensitivity due to the emissivity normalized nature of phase channel [11].

\section{B. Spectral Domain Optical Coherence Tomography}

Fig. 1(b) depicts a schematic of the developed OCT system. OCT laser is a broadband source of $30 \mathrm{~mW}$ superluminiscent diode (SLD; Exalos) centered at wavelength of $1315 \mathrm{~nm}$ and bandwidth of approximately $110 \mathrm{~nm}$. Spectrometer is a 2048pixel high speed camera with line scan/acquisition rate of 147 $\mathrm{kHz}$. The theoretical axial resolution of the system is approximately $4.5 \mu \mathrm{m}$ in tooth and the theoretical lateral resolution is $10 \mu \mathrm{m}$. The experimental axial resolution in air and tissue were measured as 12 and $8.6 \mu \mathrm{m}$, respectively. Optical power at sample is $\sim 7 \mathrm{~mW}$ and the signal-to-noise (SNR) of the built OCT system is $>100 \mathrm{~dB}$. The measured imaging depth of the system is $5 \mathrm{~mm}$ in air. The OCT beam in scanned along the smooth surface of the tooth using the two galvo scanners (GS) and performs the inverse Fourier transform operation on the Fourier-domain signals registered by the spectrometer, thus creating a 3D tomographic image of early caries. For OCT, the backscattered light in a form of integrated en-face images are measured and displayed.

\section{Controlled Demineralization Protocol}

Following the bio- and laser safety regulations at York University, anonymous extracted human teeth were collected from local oral surgeons and teeth with no visible stains or white spot lesions were selected for the study. In controlled demineralization of dental samples, a lactic acid-based solution is prepared. The solution is an acidified gel, consisting of $0.1 \mathrm{M}$ lactic acid and $0.1 \mathrm{M} \mathrm{NaOH}$ which is gelled with addition of $6 \% \mathrm{w} / \mathrm{v}$ hydroxyethylcellulose [10][11]. As such, teeth with no visible defect are selected and the surface of the tooth is covered by nail polish except for a rectangular treatment window. Sample is then submerged upside down in the acidic gel for certain number of days. Finally, sample is removed from the gel, and the nail polish is removed for imaging.

\section{Data Processing and Analysis}

To quantify the detection threshold of TPLI and OCT in progression of early caries, the thermophotonic phase images at

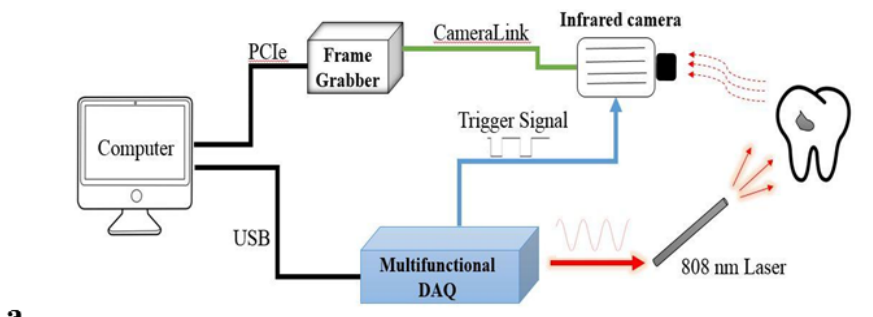

$\mathbf{a}$

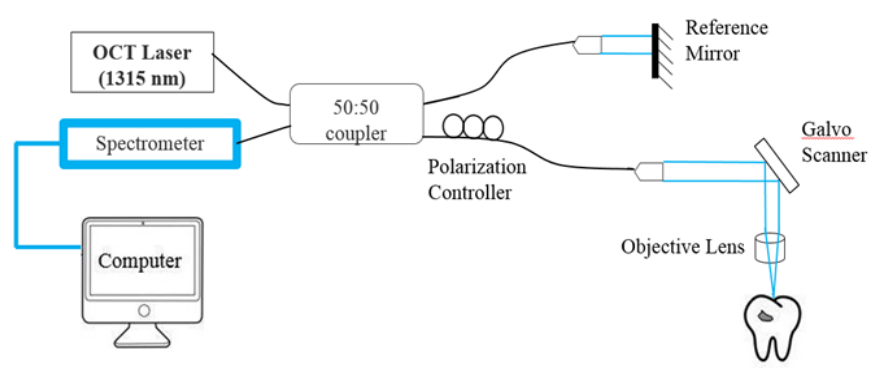

$\mathbf{b}$

Figure 1. Schematic representation of (a) TPLI system and (b) spectraldomain OCT system.

$2 \mathrm{~Hz}$ and integrated en-face images derived from OCT were normalized [11]. For TPLI phase images, the pixel values were normalized by subtracting the phase value of pixels by the average pixel value of an intact reference area from the same image. For integrated en-face images, normalization was performed by dividing the amplitude values of pixels by the average amplitude values of an intact reference area from the same image. To compare and monitor the progression of artificial tooth demineralization in OCT and TPLI, the normalized phase and integrated en-face images were averaged in the regions of interest and the standard deviation of pixel values was calculated.

\section{RESULTS AND DISCUSSION}

Detection threshold is an important parameter of a diagnostic modality, i.e. how early the diagnostic system can detect caries.

\section{A. Detection Threshold of OCT and TPLI}

To this end, a time-dependent controlled demineralization procedure was followed on smooth surface of a tooth sample. Fig. 2(a) shows the visual photograph of the sample and the dashed rectangle shows the location of the treatment window.

In integrated en-face OCT (Fig. 2(b)-(l)), it can be observed that as the treatment time increases, the contrast between the treatment window and the intact region gradually increases as a result of progression of artificially-induced early caries which results in enhancement of light scattering. The maximum contrast occurs at day 15 of demineralization. Similarly, visual inspection of TPLI phase images (Fig. 3(a)(k)) shows that TPLI starts to show very small change in contrast with progression of early caries starting from day 1 of demineralization. This change becomes fully visible at day 2 of demineralization. While the contrast from day 2 to day 10 

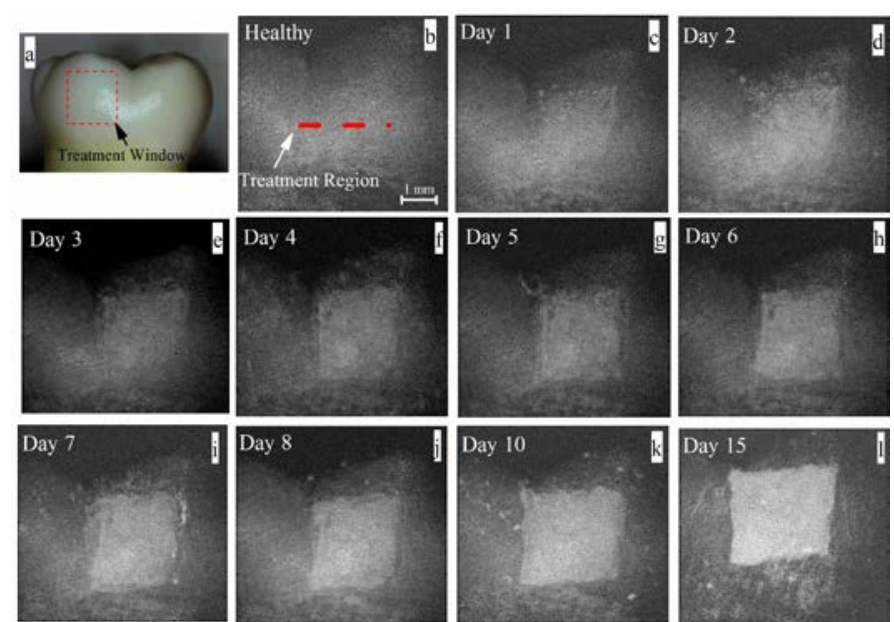

Figure 2. Image of the sample (a) before demineralization and after (b) 0 days, (c) 1 day, (d) 2 days, (e) 3 days (f) 4 days (g) 5 days (h) 6 days (i) 7 days (j) 8 days (k) 10 days, and (h) 15 days of demineralization

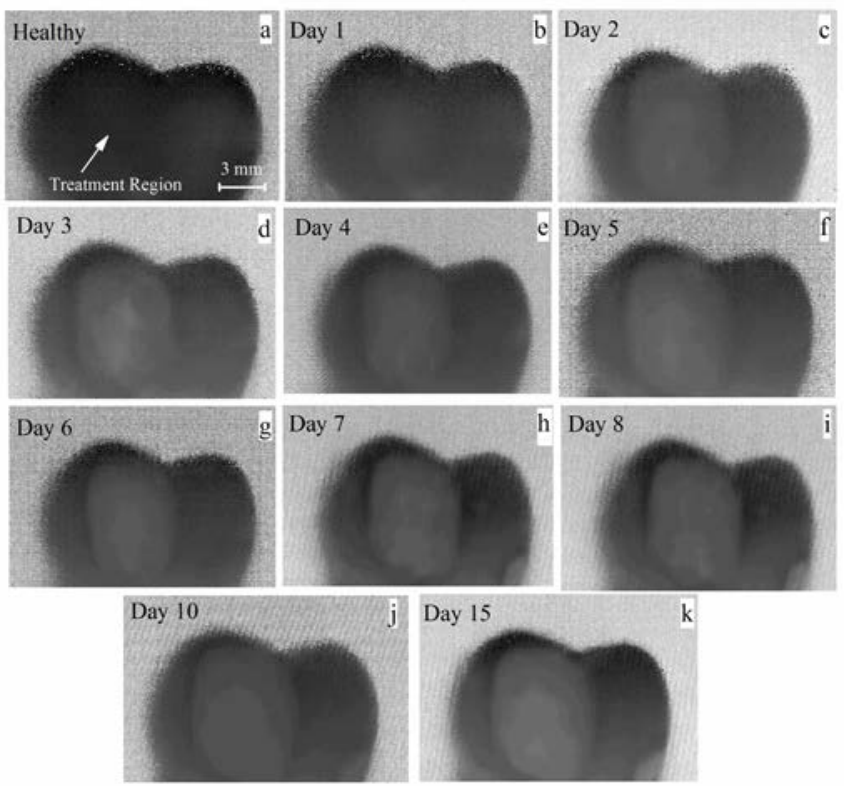

Figure 3. TPLI phase images at 2-Hz modulation frequency at (a) 0 days, (b) 1 day, (c) 2 days, (d) 3 days (e) 4 days (f) 5 days (g) 6 days (h) 7 days (i) 8 days (j) 10 days, and (k) 15 days of demineralization

increases gradually, at day 15 of demineralization the maximum contrast occurs. In addition to visual observations, statistical analysis was conducted on images of Fig. 2 and Fig. 3. The bar plots of Fig. 4 confirm the superiority of detection threshold of TPLI over OCT. In integrated en-face OCT bar plots, it can be seen that in each demineralization step, the system tends to show higher standard deviation compared to the bar plots of TPLI. The relatively large standard deviation present in OCT is due to its non-specific nature of light scattering mechanism. It is observed that with progression of artificially-induced early caries, there are gradual enhancements in average integrated intensities. However, there also exists some instability in the average integrated intensity values between the demineralization steps of day 4 to 10 . Consequently, it can be concluded that after application of demineralization for 15 days or more, OCT could differentiate between healthy and early carious lesions as the standard deviation of treatment steps up to day 10 contain intensity values which lie within the standard deviation of the healthy region. Thus, at 15-day of treatment step the detection threshold of the system can be defined. Bar plots of TPLI show an increase in the average phase values between each treatment interval. Due to smaller standard deviation of average phase value obtained from the healthy region of the sample, the detection threshold of TPLI can be distinguished at day 2 of demineralization. More comprehensive comparison between TPLI and OCT is tabulated in Table 1. From these comparison parameters it can be concluded that TPLI is an optimal choice for detection of early dental caries.
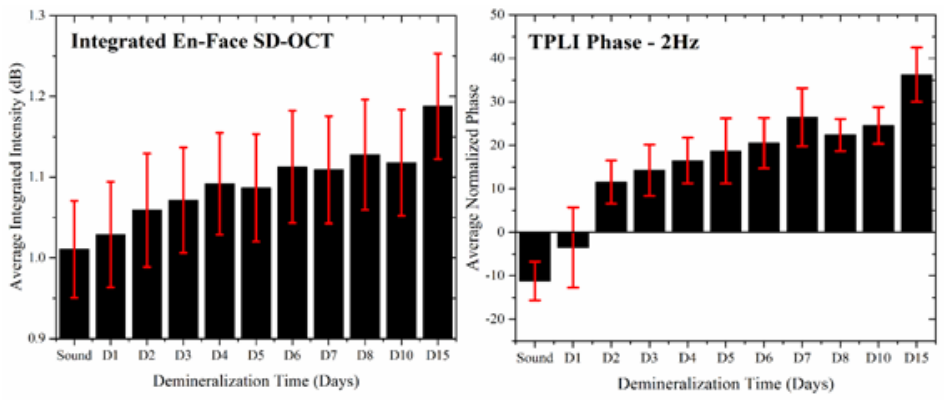

Figure 4. Bar plots of (left) spectral-domain optical coherence tomography (OCT) and thermophotonic lock-in imaging (TPLI) (right) with their standard deviations.

\begin{tabular}{ccc}
\hline \multicolumn{2}{c}{ TABLE 1. SUMMARY OF COMPARISON BETWEEN TPLI AND OCT } \\
\hline Parameters & TPLI & OCT \\
\hline Source of Contrast & Absorption & Scattering \\
\hline Imaging Depth & few mm-cm & $1-2 \mathrm{~mm}$ \\
Resolution & Low $(\sim 100 \mu \mathrm{m})$ & High $(\sim 10 \mu \mathrm{m})$ \\
& & \\
Field of view & Wide & Small \\
(whole tooth) & \\
Detection Sensitivity & Very High & Low \\
\hline Detection Threshold & 2 Days of Demin & 15 Days of Demin \\
\hline 3D Imaging & No & Yes \\
\hline Clinical Translation & Difficult & Straight Forward \\
\hline Cost $(\$)$ & $\$$ & $\$ \$$ \\
\hline
\end{tabular}

\section{CONCLUSION}

In this study, we present a study that quantifies the detection threshold between spectral domain optical coherence tomography and thermophotonic lock-in imaging in detection of early dental caries. These systems used for diagnosis of early caries and their progression would significantly improve caries management decisions with respect to preventive care. Results suggest that the detection threshold for detection of early dental caries were found to be better in TPLI images compared to OCT due to its light absorption contrast mechanism compared to light scattering contrast mechanism in OCT. 


\section{REFERENCES}

[1] R.A. Bagramian, F. Garcia-Godoy, and A.R. Volpe, "The global increase in dental caries. A pending public health crisis,” Am. J. Dent., vol. 22, no. 1, pp. 3-8, 2009.

[2] M. Mohanraj, V. R. Prabhu, R. Senthil, "Diagnostic methods for early detection of dental caries- A review” Int. J. Pedod. Rehabil. Vol. 1, no. 1, pp. 29-36, 2016.

[3] N. Garg, and A. Garg, Text Book of Operative Dentistry, 2nd ed. Jp Medical Ltd, Faridabad, Haryana, Indian (2012).

[4] Y.S. Hsieh, Y.C. Ho, S.Y. Lee, C.-C. Chuang, J. Tsai, K.-F. Lin, and C.W. Sun, "Dental Optical Coherence Tomography," Sensors, vol. 13, no. 7, pp. 8928-8949, 2013.

[5] M.T. Tsai et al. "Effective indicators for diagnosis of oral cancer using optical coherence tomography." Optics express, vol.16, no. 20, pp. 15847-15862, 2008.

[6] Y. Shimada, A. Sadr, M. Burrow, J. Tagami, N. Ozawa, and Y. Sumi, "Validation of swept-source optical coherence tomography (SS-OCT) for the diagnosis of occlusal caries," J. Dent., vol. 38, no. 8, pp. 655665, 2010.
[7] O. Breitenstein, W. Wilhelm, and M. Langenkamp, Lock-in thermography: Basics and use for evaluating electronic devices and materials. vol. 10. Springer Science \& Business Media, 2010.

[8] Y.-K. An, J. Kim, and H. Sohn, "Laser lock-in thermography for detection of surface-breaking fatigue cracks on uncoated steel structures,” NDT\&E Int., vol. 65, pp. 54-63, 2014.

[9] N. Tabatabaei, A. Mandelis, and B. Amaechi, "Thermophotonic lock-in imaging of early demineralized and carious lesions in human teeth,” J. Biomed. Opt., vol. 16, no. 7, pp. 071402-071402-10, 2011.

[10] A. Ojaghi, A. Parkhimchyk, and N. Tabatabaei, "First step toward translation of thermophotonic lock-in imaging to dentistry as an early caries detection technology,” J. Biomed. Opt., vol. 21, no. 9, pp. 096003-096003, 2016.

[11] N. Tabatabaei, A. Mandelis, M. Dehghany, K. Michaelian, and B. Amaechi, "On the sensitivity of thermophotonic lock-in imaging and polarized Raman spectroscopy to early dental caries diagnosis,” J. Biomed. Opt., vol. 17, no. 2, pp. 0250021-0250025, 2012. 